Pacific Journal of Mathematics

TRIODIC HOMOGENEOUS CONTINUA 


\title{
ATRIODIC HOMOGENEOUS CONTINUA
}

\author{
Charles L. Hagopian \\ Dedicated to Professor F. Burton Jones
}

\begin{abstract}
In answer to a question of T. Mackowiak and E. D. Tymchatyn [20] we prove that every atriodic homogeneous continuum is 1-dimensional. This is accomplished by showing that every atriodic homogeneous continuum that is not a solenoid and has a decomposable subcontinuum admits a continuous decomposition to a solenoid and that all elements of this decomposition are homeomorphic tree-like hereditarily indecomposable homogeneous continua. It follows from this decomposition theorem that every tree-like atriodic homogeneous continuum is hereditarily indecomposable. This decomposition theorem also provides another proof of the author's theorem [11] that every indecomposable homogeneous plane continuum is hereditarily indecomposable.
\end{abstract}

1. Introduction. A space is homogeneous if for each pair $p, q$ of its points there is a homeomorphism of the space onto itself that takes $p$ to $q$. A continuum is a compact connected nondegenerate metric space. A continuum $M$ is a triod if $M$ has a subcontinuum $H$ such that $M \backslash H$ is the union of three nonempty disjoint opens sets. When a continuum does not contain a triod it is atriodic. A continuum $M$ is tree-like if for each positive number $\varepsilon$ there is an open covering of $M$ with mesh less than $\varepsilon$ whose nerve is a tree. A continuum is decomposable if it is the union of two of its proper subcontinua; otherwise, it is indecomposable. When a continuum does not have a decomposable subcontinuum it is hereditarily indecomposable. Note that every hereditarily indecomposable continuum is atriodic.

In $1951 \mathrm{R}$. H. Bing [2] proved that every finite-dimensional hereditarily indecomposable homogeneous continuum is 1-dimensional. Recently J. T. Rogers, Jr. [25] proved that every hereditarily indecomposable homogeneous continuum is tree-like and, therefore, 1-dimensional. Mackowiak and Tymchatyn [20, Theorem 13.4] proved that every finite-dimensional atriodic homogeneous continuum is 1-dimensional. In $\$ 13$ of [20], Mackowiak and Tymchatyn asked if every atriodic homogeneous continuum is 1-dimensional. Corollary 1 of $\$ 4$ (below) answers this question in the affirmative.

Our arguments involve a decomposition theory for homogeneous continua that was originated in 1951 by F. B. Jones [15]. Recently Rogers [24] surveyed this area and presented a general decomposition theory for homogeneous spaces. Theorem 2 of $\S 4$ (below) solves a problem of Jones' 
that Rogers [24, page 142] called the outstanding problem in decompositions of homogeneous continua.

Mackowiak and Tymchatyn [20, Theorem 14.7] proved that every decomposable atriodic homogeneous continuum that is not a simple closed curve has a continuous decomposition to a circle and that the elements of this decomposition are homeomorphic indecomposable homogeneous continua. In $\$ 14$ of [20] they asked if every atriodic homogeneous continuum that is not a solenoid and has a decomposable subcontinuum admits a continuous decomposition to a solenoid such that all elements of the decomposition are homeomorphic tree-like hereditarily indecomposable homogeneous continuum. Theorem 2 of $\$ 4$ (below) answers this question in the affirmative.

Bing [4, Theorem 10] proved that no tree-like atriodic homogeneous continuum contains an arc. Mackowiak and Tymchatyn [20, Theorem 14.8] generalized Bing's theorem by proving that no tree-like atriodic homogeneous continuum has a hereditarily decomposable subcontinuum. According to Corollary 2 of $\$ 4$ (below), no tree-like atriodic homogeneous continuum has a decomposable subcontinuum.

The known examples of atriodic homogeneous continua are the solenoids [12], the pseudo-arc [1], and the solenoids of pseudo-arcs [23] [13]. By Rogers' theorem [25] and Theorem 2 of $\$ 4$ (below), if every tree-like homogeneous continuum is a pseudo-arc, then there are no other examples of atriodic homogeneous continua. Unfortunately, it is not known whether the pseudo-arc is the only tree-like continuum that is homogeneous. For additional information and unsolved problems involving 1-dimensional homogeneous continua see C. E. Burgess' expository article [7].

2. More definitions and related results. A chain is a finite collection $\left\{L_{i}: 1 \leq i \leq n\right\}$ of open sets such that $L_{l} \cap L_{j} \neq \varnothing$ if and only if $|i-j| \leq 1$. If $L_{1}$ also intersects $L_{n}$ the collection is called a circular chain. Each $L_{i}$ is called a link. A chain (circular chain) is called an $\varepsilon$-chain ( $\varepsilon$-circular chain) if each of its links has diameter less than $\varepsilon$. A continuum is arc-like (circle-like) if for each positive number $\varepsilon$, it can be covered by an $\varepsilon$-chain ( $\varepsilon$-circluar chain). Bing [1] [3] proved that a continuum is a pseudo-arc if and only if it is homogeneous and arc-like.

A continuum is a solenoid if it is homeomorphic to an inverse limit of circles with covering maps as the bonding maps. Note that simple closed curves are solenoids. The author [12] proved that a continuum $M$ is a solenoid if and only if $M$ is homogeneous and every proper subcontinuum of $M$ is an arc. Rogers [24, Theorem 3] proved that every atriodic 
homogeneous 1-dimensional continuum that contains an arc is a solenoid. In [20, Theorem 14.8], Mackowiak and Tymchatyn generalized these results by showing that every atriodic homogeneous continuum that contains a hereditarily decomposable continuum is a solenoid.

A continuum $M$ is a solenoid of pseudo-arcs if $M$ is circle-like and there exists a continuous decomposition $\mathscr{D}$ of $M$ to a solenoid such that each element of $\mathscr{Q}$ is a pseudo-arc. In 1959 Bing and Jones [5] constructed the circle of pseudo-arcs. Rogers [23] used this continuum to construct a solenoid of pseudo-arcs for each solenoid. The author and Rogers [13] proved that every circle-like homogeneous continuum is either a solenoid, a pseudo-arc, or a solenoid of pseudo-arcs.

Following K. Kuratowski [18] we define a continuum $M$ to be of type $\lambda$ if $M$ is irreducible and every indecomposable continuum in $M$ is a continuum of condensation. Type $\lambda$ continua are studied by E. S. Thomas in [26]. There they are called continua of type $A^{\prime}$.

If a continuum $M$ is of type $\lambda$, then $M$ admits a unique monotone upper semi-continuous decomposition of such that $M / \mathcal{D}$ is an arc and each element of $\mathscr{D}$ has a void interior relative to $M[19$, Theorem 3, page 216] [26, Theorem 10, page 15]. We shall refer to $\mathscr{D}$ as simply the decomposition of $M$.

3. Preliminaries. Throughout this section $M$ is an atriodic homogeneous continuum with metric $\rho$.

Let $\varepsilon$ be a positive number. A homeomorphism $h$ of $M$ onto $M$ is called an $\varepsilon$-homeomorphism if $\rho(x, h(x))<\varepsilon$ for each point $x$ of $M$.

Notation. Let $x$ be a point of $M$. We denote $\{y \in M$ : an $\varepsilon$-homeomorphism of $M$ onto $M$ takes $x$ to $y$ \} by $W(x, \varepsilon)$. Let $X$ be a subset of $M$. We denote $\cup\{W(x, \varepsilon): x \in X\}$ by $W(X, \varepsilon)$.

LEMMA 1. For every positive number $\varepsilon$ and every point $x$ of $M$, the set $W(x, \varepsilon)$ is open in $M$.

Proof. Lemma 1 follows from a short argument [10, Lemma 4, proof] involving E. G. Effros' topological transformation group theorem [8, Theorem 2.1].

A continuum is unicoherent provided that if it is the union of two subcontinuum $H$ and $K$, then $H \cap K$ is connected.

LEMMA 2. Every proper subcontinuum of $M$ is unicoherent [20, Theorem 13.8]. 
In the remainder of this section we assume there is a continuum $E$ of type $\lambda$ in $M$. Let $k: E \rightarrow[0,1]$ be a quotient map associated with the decomposition of $E$. We call $k^{-1}(0)$ and $k^{-1}(1)$ the end sets of $E$.

LEMMA 3. Let $Y$ be an element of the decomposition of $E$ distinct from $k^{-1}(0)$ and $k^{-1}(1)$. Let $F$ be a type $\lambda$ subcontinuum of $M$ with ends $T$ and $V$, and let $U$ be an element of the decomposition of $F$ distinct from $T$ and $V$. Suppose $h$ is a homeomorphism of $M$ onto $M$ such that $U \cap h[Y] \neq \varnothing$ and $U \cap h\left[k^{-1}(0) \cup k^{-1}(1)\right]=\varnothing=h[Y] \cap(T \cup V)$. Then $h[Y]=U$.

Proof. Lemma 3 follows from the argument given in paragraphs 9 through 11 in the proof of Theorem 1 of [10].

A subcontinuum $F$ of $M$ is called an extension of $E$ away from $k^{-1}(0)$ if $F$ is a continuum of type $\lambda$ that contains $E$ and has $k^{-1}(0)$ as an end set.

Notation. We denote the set of all extensions of $E$ away from $k^{-1}(0)$ by $\mathcal{E}\left(k^{-1}(0), E\right)$.

LEMMA 4. The set $\mathcal{E}\left(k^{-1}(0), E\right)$ is linearly ordered by inclusion and does not have a maximal element [11, Lemma 4].

LEMMA 5. The decomposition of each continuum of type $\lambda$ in $M$ is continuous [11, Lemma 5].

A continuum $H$ in $E$ is an essential subcontinuum of $E$ if $H$ intersects more than one element of the decomposition of $E$.

LEMMA 6. If $H$ is an essential subcontinuum of $E$, then $H$ is a continuum of type $\lambda$ and every element of the decomposition of $H$ is an element of the decomposition of $E$.

Proof. Lemma 6 follows from Lemma 5 and the irreducibility properties of $E$ [26, Theorem 8, page 14].

LeMma 7. Suppose $F$ is a continuum of type $\lambda$ in $M$ such that $E \backslash F \neq$ $\varnothing \neq F \backslash E$. Suppose $F \cap k^{-1}(r) \neq \varnothing$ for some number $r(0 \leq r \leq 1)$. Then $k^{-1}(r)$ is an element of the decomposition of $F$.

Proof. By Lemma 4, there is a continuum $H$ of type $\lambda$ in $M$ such that

(1) $E$ is an essential subcontinuum of $H$ that misses both end sets of $H$. 
Let $I$ be a continuum of type $\lambda$ in $M$ such that $F$ is an essential subcontinuum of $I$ that misses both end sets of $I$.

Observe that

(2) $k^{-1}(r) \cap(I \backslash F)=\varnothing$.

To see this note that since $F \backslash E \neq \varnothing$, the continuum $F$ is not in $k^{-1}(r)$. Since $M$ is atriodic, it follows from (1) that there is a point $x$ of $F$ in $H \backslash k^{-1}(r)$. Let $J$ be the continuum of type $\lambda$ in $H$ such that $x$ belongs to one end set of $J$ and the other end set of $J$ is $k^{-1}(r)$. By Lemma 2, $F \cap J$ is a subcontinuum of $J$.

Suppose that (2) is false. Then $F \cap J$ does not contain $k^{-1}(r)$. Hence $F \cap J$ is a proper subcontinuum of $J$. Since $x \in F \cap J$ and $F \cap J$ intersects $k^{-1}(r)$, this contradicts the fact that $J$ is irreducible between $x$ and $k^{-1}(r)$. Hence (2) is true.

Since $M$ is atriodic and $F \cap k^{-1}(r) \neq \varnothing$, it follows from (2) that $k^{-1}(r) \subset F$. Therefore, since $M$ is atriodic and $E \backslash F \neq \varnothing$, there is a point $y$ of $E \backslash k^{-1}(r)$ in $I \backslash F$.

Let $K$ be the continuum of type $\lambda$ in $E$ such that $y$ belongs to one end set of $K$ and the other end set of $K$ is $k^{-1}(r)$. Let $L$ be a subcontinuum of $I$ that is irreducible between $y$ and $k^{-1}(r)$. It follows from Lemma 2 and the irreducibility of $K$ and $L$ that $K=K \cap L=L$. Hence $L$ is a continuum of type $\lambda$ and $k^{-1}(r)$ is an element of the decomposition of $L$. Since $k^{-1}(r) \subset F$ and $y \in I \backslash F$, the continuum $L$ is an essential subcontinuum of $I$. By Lemma $6, k^{-1}(r)$ is an element of the decomposition of $I$. Since $F$ is an essential subcontinuum of $I$ and $k^{-1}(r) \subset F$, it follows from Lemma 6 that $k^{-1}(r)$ is an element of the decomposition of $F$.

LEMMA 8. Suppose $F$ is a continuum of type $\lambda$ in $M$ that intersects $E$ and misses either $k^{-1}(0)$ or $k^{-1}(1)$. Then $\cup\left(\mathcal{E}\left(k^{-1}(0), E\right) \cup \mathcal{E}\left(k^{-1}(1), E\right)\right)$ contains $F$.

Proof. The conclusion follows immediately if $E$ contains $F$. Therefore we assume that $F \not \subset E$. Assume without loss of generality that $F$ misses $k^{-1}(0)$. By Lemma 7, for each number $r(0<r \leq 1)$ if $k^{-1}(r)$ intersects an element $Y$ of the decomposition of $F$, then $k^{-1}(r)=Y$. Hence the union Q 2 of the decompositions of $E$ and $F$ is a monotone continuous (Lemma 5) decomposition of the continuum $E \cup F$. Each element of 2 has void interior relative to $E \cup F$. The quotient space $(E \cup F) / \mathscr{Q}$ is the union of two arcs. Since $M$ is atriodic, $(E \cup F) / \mathscr{Q}$ is atriodic. Moreover $(E \cup F) / \mathcal{Q}$ is not a simple closed curve since $F$ misses $k^{-1}(0)$. It follows from Lemma 2 that $(E \cup F) / \mathscr{D}$ does not contain a simple closed 
curve. Thus $(E \cup F) / \mathcal{Q}$ is an arc and $E \cup F$ is a continuum of type $\lambda$. Furthermore $k^{-1}(0)$ is an end set of $E \cup F$. Hence $E \cup F$ belongs to $\mathcal{E}\left(k^{-1}(0), E\right)$. This completes the proof of Lemma 8 .

LEMMA 9. Every element of the decomposition of $E$ is homogeneous.

Proof. Lemma 9 follows from paragraphs 5 through 12 in the proof of Theorem 1 of [10].

LemMA 10. Suppose $N$ is an indecomposable subcontinuum of $M$ that contains $E$. Then $N$ contains $\cup \mathcal{E}\left(k^{-1}(0), E\right)$.

Proof. Assume $N$ does not contain $\cup \mathcal{E}\left(k^{-1}(0), E\right)$. Let $F$ be an element of $\mathcal{E}\left(k^{-1}(0), E\right)$ that intersects $M \backslash N$. Let $A$ be the composant of $N$ that contains $E$. Let $Y$ be the end set of $F$ opposite $k^{-1}(0)$. It follows from Lemma 2 and the irreducibility of $F$ that $N \cap Y=\varnothing$. Let $\varepsilon=$ $\rho(N, Y)$. By Lemma 1 , there exist two $\varepsilon$-homomorphisms $f$ and $g$ of $M$ onto $M$ and two composants $B$ and $C$ of $N$ distinct from $A$ such that $f[F] \cap B \neq \varnothing \neq g[F] \cap C$. By Lemma $2, F, f[F]$, and $g[F]$ are disjoint. Since $f$ and $g$ are $\varepsilon$-homeomorphisms, $M \backslash N$ contains $f[Y] \cup g[Y]$. Hence $N \cup F \cup f[F] \cup g[F]$ is a triod. This contradicts the assumption that $M$ is atriodic. Therefore $N$ contains $\cup \mathcal{E}\left(k^{-1}(0), E\right)$.

Notation. Let $X$ be a subset of $M$. We denote the boundary of $X$ and the closure of $X$ in $M$ by $\mathrm{Bd} X$ and $\mathrm{Cl} X$, respectively.

LEMMA 11. If $N$ is an indecomposable subcontinuum of $M$ that contains $E$, then $\mathrm{Cl} \cup \mathcal{E}\left(k^{-1}(0), E\right)$ is an indecomposable subcontinuum of $N$.

Proof. By Lemma 10, $\mathrm{Cl} \cup \mathcal{E}\left(k^{-1}(0), E\right)$ is a subcontinuum of $N$. The argument given in paragraphs 2 through 11 in the proof of Lemma 6 of [11] proves that $\mathrm{Cl} \cup \mathcal{E}\left(k^{-1}(0), E\right)$ is indecomposable.

Suppose $\mathcal{L}=\left\{L_{i}: 1 \leq i \leq 5\right\}$ is a 5 -linked chain in $M$.

Notation. We denote the 3-linked subchain $\left\{L_{i}: 2 \leq i \leq 4\right\}$ of $\mathscr{E}$ by $\mathcal{L}^{\prime}$.

The chain $\mathcal{L}$ is free if $\operatorname{Bd}\left(L_{1} \cup L_{5}\right) \backslash \mathrm{Cl} \cup \mathcal{L}^{\prime}$ contains $\mathrm{Bd} \cup \mathcal{L}$.

The chain $\mathcal{L}$ is normal if $\mathrm{Cl} L_{i} \cap \mathrm{Cl} L_{j}=\varnothing$ whenever $|i-j|>1$.

The continuum $E$ runs straight through $\mathcal{L}$ provided

(1) $E \subset \cup \mathcal{L}$,

(2) $k^{-1}(0) \subset L_{1} \backslash L_{2}$, 
(3) $k^{-1}(1) \subset L_{5} \backslash L_{4}$,

(4) if $0 \leq r \leq 1$ and $k^{-1}(r) \cap \mathrm{Bd} L_{i} \neq \varnothing$, then $k^{-1}(r) \subset \mathrm{Bd} L_{i}$, and

(5) if $0 \leq r<t \leq 1$ and $k^{-1}(r) \cup k^{-1}(t) \subset L_{i}$, then $k^{-1}[[r, t]] \subset L_{i}$.

The chain $\mathcal{E}$ is regular if for each component $K$ of $\cup \mathcal{L}^{\prime}$, the set $\mathrm{Cl} K$ is a continuum of type $\lambda$ that runs straight through $\mathcal{L}$.

A chain $\left\{P_{l}: 1 \leq i \leq 5\right\}$ is an ordered refinement of $\varrho$ if for each $i$, the link $L_{l}$ contains $P_{l}$.

LEMMA 12. Suppose $E$ runs straight through a normal regular chain $\mathcal{L}=\left\{L_{i}: 1 \leq i \leq 5\right\}$. Suppose $k^{-1}(0) \subset L_{1} \backslash \mathrm{Cl} L_{2}$ and $k^{-1}(1) \subset L_{5} \backslash \mathrm{Cl} L_{4}$. Then $E$ runs straight through a free normal regular ordered refinement of $\mathrm{L}$.

Proof. Let $A$ and $B$ denote the open sets $L_{1} \backslash \mathrm{Cl} L_{2}$ and $L_{5} \backslash \mathrm{Cl} L_{4}$, respectively. Since $\mathcal{L}$ is regular, $E \backslash(A \cup B)$ is an essential subcontinuum of $E$. Since $M$ is atriodic, $E \backslash(A \cup B)$ is a component of $M \backslash(A \cup B)$. Since no component of $M \backslash(A \cup B)$ intersects both $E \backslash(A \cup B)$ and $M \backslash \cup \mathcal{L}$, there exist disjoint open sets $C$ and $D$ in $M$ such that (1) $C$ contains $E \backslash(A \cup B)$, (2) $D$ contains $M \backslash \cup \mathcal{L}$, and (3) $C \cup D$ contains $M \backslash(A \cup B)$ [22, Theorem 44, page 15].

Let $P_{1}=L_{1}$ and $P_{5}=L_{5}$. For $i=2,3$, and 4 , let $P_{l}=C \cap L_{l}$. The chain $\mathscr{P}=\left\{P_{i}: 1 \leq i \leq 5\right\}$ is a free normal regular ordered refinement of $\mathcal{L}$. Note that $E$ runs straight through $\mathcal{P}$.

LEMMA 13. Suppose $\varepsilon$ is a positive number, $A$ is a closed set that misses $E$, and $B$ is an open set that contains $k^{-1}(0) \cup k^{-1}(1)$. Then $E$ runs straight through a free normal regular chain $\mathscr{P}=\left\{P_{i}: 1 \leq i \leq 5\right\}$ in $M \backslash A$ with the property that $B$ contains $\mathrm{Cl}\left(P_{1} \cup P_{5}\right)$ and for each component $K$ of $\cup \mathcal{P}^{\prime}$ there is an $\varepsilon$-homeomorphism $h$ of $M$ onto $M$ such that $\mathrm{Cl} K$ is an essential subcontinuum of $h[E]$.

Proof. Let $\left\{x_{i}: 0 \leq i \leq 15\right\}$ be a set of numbers such that $x_{0}=0$, $x_{15}=1, x_{i}<x_{i+1}$ for each $i(0 \leq i \leq 14)$, and $k^{-1}\left[\left[0, x_{3}\right] \cup\left[x_{12}, 1\right]\right] \subset B$. Let $\delta$ be a positive number less than $\varepsilon, \rho(A, E), \rho\left(M \backslash B, k^{-1}\left[\left[0, x_{3}\right] \cup\right.\right.$ $\left.\left.\left[x_{12}, 1\right]\right]\right)$, and the minimum of $\left\{\frac{1}{2} \rho\left(k^{-1}\left[\left[0, x_{i}\right]\right], k^{-1}\left[\left[x_{l+1}, 1\right]\right]\right): 1 \leq i \leq\right.$ $13\}$.

For each integer $i \quad(1 \leq i \leq 5)$, let $L_{l}$ be the open set $W\left(k^{-1}\left[\left[x_{3 i-3}, x_{3 l}\right]\right], \delta\right)($ Lemma 1$)$. Let $\mathcal{L}=\left\{L_{i}: 1 \leq i \leq 5\right\}$. Note that $\mathcal{L}$ is a normal chain in $M \backslash A$.

Next we prove that $e$ is regular. To accomplish this let $h$ be a $\delta$-homeomorphism of $M$ onto $M$. 
Note that

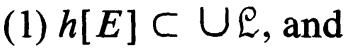

(2) $h\left[k^{-1}(0)\right] \subset L_{1} \backslash \mathrm{Cl} L_{2}$ and $h\left[k^{-1}(1)\right] \subset L_{5} \backslash \mathrm{Cl} L_{4}$.

For each number $r(0 \leq r \leq 1)$ and each integer $i(1 \leq i \leq 5)$

(3) if $h\left[k^{-1}(r)\right] \cap L_{i} \neq \varnothing$, then $h\left[k^{-1}(r)\right] \subset L_{i}$.

To prove (3) assume for some numbers $r$ and $i, h\left[k^{-1}(r)\right]$ intersects both $L_{i}$ and $M \backslash L_{i}$. It follows from the definition of $\delta$ that $r \in$ $\left[x_{2}, x_{13}\right] \backslash\left[x_{3 i-3}, x_{3 i}\right]$. There exist a number $s$ in $\left[x_{3 i-3}, x_{3 i}\right] \cap\left[x_{2}, x_{13}\right]$ and a $\delta$-homeomorphism $g$ of $M$ onto $M$ such that $g\left[k^{-1}(s)\right] \cap h\left[k^{-1}(r)\right]$ $\neq \varnothing$. By Lemma 3, $g\left[k^{-1}(s)\right]=h\left[k^{-1}(r)\right]$. This contradicts the fact that $L_{i}$ contains $g\left[k^{-1}(s)\right]$. Hence (3) holds.

For each number $r(0 \leq r \leq 1)$

(4) if $h\left[k^{-1}(r)\right] \cap \mathrm{Bd} L_{i} \neq \varnothing$, then $h\left[k^{-1}(r)\right] \subset \mathrm{Bd} L_{i}$.

To see this let $p$ be a point of $h\left[k^{-1}(r)\right] \cap \mathrm{Bd} L_{i}$ and assume that Bd $L_{i}$ misses a point $q$ of $h\left[k^{-1}(r)\right]$. By (3), $q \notin L_{i}$. Let $\mu=\rho\left(q, L_{i}\right)$. By Lemma 1 , there exists a $\mu$-homeomorphism $f$ of $M$ onto $M$ such that $f h$ is a $\delta$-homeomorphism and $f(p) \in L_{i}$. It follows from the argument for (3) that $f h\left[k^{-1}(r)\right] \subset L_{i}$. This contradicts the fact that $f(q) \notin L_{i}$. Hence (4) holds.

Note that

(5) if $0 \leq r<t \leq 1$ and $k^{-1}(r) \cup k^{-1}(t) \subset L_{i}$, then $k^{-1}[[r, t]] \subset L_{i}$.

To see this assume the contrary. By (1), (2), and (3), there exist numbers $r, s$, and $t(0 \leq r<s<t \leq 1)$ and an integer $i(1 \leq i \leq 4)$ such that $h\left[k^{-1}(s)\right] \subset L_{i}$ and $h\left[k^{-1}(r) \cup k^{-1}(t)\right] \subset L_{i+1} \backslash L_{i}$. Let $u$ be a number in $\left[x_{3 i-3}, x_{3 i}\right]$ and $g$ be a $\delta$-homeomorphism of $M$ onto $M$ such that $g\left[k^{-1}(u)\right] \cap h\left[k^{-1}(s)\right] \neq \varnothing$. Since $M$ is atriodic and $\left.g\left[k^{-1}\left(0, x_{3 i}\right)\right]\right]$ misses $L_{i+1} \backslash L_{i}$, it follows that $g\left[k^{-1}\left[0, x_{3 i}\right]\right] \subset h\left[k^{-1}[[r, t]]\right.$. This contradicts the definition of $\delta$. Hence (5) holds.

It follows from (1), (2), (4), and (5) that

(6) $h[E]$ runs straight through $\mathcal{L}$.

Let $K$ be a component of $\cup \mathcal{L}^{\prime}$. Let $h$ be a $\delta$-homeomorphism of $M$ onto $M$ such that $K \cap h[E] \neq \varnothing$. Since $M$ is atriodic, it follows from (6) and Lemma 6 that $\mathrm{Cl} K$ is an essential subcontinuum of $h[E]$ that runs straight through $\mathcal{L}$. Hence $\mathcal{L}$ is regular.

Since $h$ in (2) and (6) can be the identity, $k^{-1}(0) \subset L_{1} \backslash \mathrm{Cl} L_{2}, k^{-1}(1)$ $\subset L_{5} \backslash \mathrm{Cl} L_{4}$, and $E$ runs straight through $\mathcal{L}$. By Lemma 12, $E$ runs straight through a free normal regular ordered refinement $\mathcal{P}=\left\{P_{i}: 1 \leq i\right.$ $\leq 5\}$ of $\mathcal{L}$.

Since $\cup \mathcal{C} \subset M \backslash A$ and $\mathrm{Cl}\left(L_{1} \cup L_{5}\right) \subset B$, it follows that $\cup \mathcal{P} \subset$ $M \backslash A$ and $\operatorname{Cl}\left(P_{1} \cup P_{5}\right) \subset B$. Let $K$ be a component of $\cup \mathcal{P}^{\prime}$. Since $\delta<\varepsilon$, 
$M$ is atriodic, and $\mathcal{P}$ is a regular ordered refinement of $\mathcal{L}$, there is an $\varepsilon$-homeomorphism $h$ of $M$ onto $M$ such that $\mathrm{Cl} K$ is an essential subcontinuum of $h[E]$. This completes the proof of Lemma 13.

Notation. Suppose $\mathscr{P}=\left\{P_{i}: 1 \leq i \leq 5\right\}$ is a regular chain. Let $\Omega(\mathscr{P})$ denote the collection $\{Z: Z$ is an element of the decomposition of the closure of a component of $\left.\cup \mathscr{P}^{\prime}\right\}$. Let $\Delta(\mathscr{P})$ be $\{Z: Z \in \Omega(\mathscr{P})$ and $\left.Z \cap \mathrm{Cl}\left(P_{1} \cup P_{5}\right)=\varnothing\right\}$. Note that since $\mathcal{P}$ is regular, $\Delta(\mathcal{P})$ is a decomposition of $(\cup \mathcal{P}) \backslash \mathrm{Cl}\left(P_{1} \cup P_{5}\right)$.

Lemma 14. Suppose $\mathscr{P}=\left\{P_{i}: 1 \leq i \leq 5\right\}$ is a free regular chain. Then the decomposition $\Delta(\mathscr{P})$ of $(\cup \mathscr{P}) \backslash \mathrm{Cl}\left(P_{1} \cup P_{5}\right)$ is continuous.

Proof. Suppose $\left\{Z_{i}\right\}$ and $\left\{z_{i}\right\}$ are sequences such that (1) $Z_{i} \in \Delta(\mathscr{P})$ and $z_{i} \in Z_{i}$ for each positive integer $i$, and (2) $\left\{z_{i}\right\}$ converges to a point $z$ of $\cup \Delta(\mathscr{P})$. Let $Z$ be the element of $\Delta(\mathscr{P})$ that contains $z$. It suffices to show that $\left\{Z_{i}\right\}$ converges to $Z$.

Let $\varepsilon$ be any positive number less than $\frac{1}{2} \rho\left(\cup \Delta(\mathcal{P}), M \backslash \cup \mathcal{P}^{\prime}\right)$. Let $K_{0}$ be the $z$-component of $\cup \mathcal{P}^{\prime}$. For each positive integer $i$, let $K_{i}$ be the $z_{i}$-component of $\cup \mathscr{P}^{\prime}$. Since $\mathcal{P}$ is regular, for each non-negative integer $i$, the end sets of $\mathrm{Cl} K_{i}$ are in $M \backslash \cup \mathcal{P}^{\prime}$. By Lemma 3, if $z_{i}$ belongs to the open set $W(z, \varepsilon)$ (Lemma 1), then there is an $\varepsilon$-homeomorphism $h$ of $M$ onto $M$ such that $h[Z]=Z_{i}$. Since $\varepsilon$ is arbitrarily small, $\left\{Z_{i}\right\}$ converges to $Z$. Therefore $\Delta(\mathcal{P})$ is continuous.

LeMma 15. Suppose $\mathscr{P}=\left\{P_{i}: 1 \leq i \leq 5\right\}$ is a free regular chain. Then there is a positive number $\varepsilon$ such that if $Z \in \Delta(\mathscr{P})$ and $h$ is an $\varepsilon$-homeomorphism of $M$ onto $M$, then $h[Z] \in \Omega(\mathscr{P})$.

Proof. Let $\varepsilon=\frac{1}{2} \rho\left(\cup \Delta(\mathscr{P}), M \backslash \cup \mathscr{P}^{\prime}\right)$. By Lemma 3, if $Z \in \Delta(\mathscr{P})$ and $h$ is an $\varepsilon$-homeomorphism of $M$ onto $M$, then $h[Z] \in \Omega(\mathscr{P})$.

LEMma 16. Suppose $\mathcal{P}=\left\{P_{i}: 1 \leq i \leq 5\right\}$ is a free regular chain. Suppose $K$ is a component of $\cup \mathcal{P}^{\prime}$ and $X$ is an end set of $\mathrm{Cl} K$. Suppose $F$ is an extension of $\mathrm{Cl} K$ away from $X$ and $\mathscr{Q}$ is the decomposition of $F$. Then no element of $\mathscr{D}$ intersects three consecutive links of $\mathcal{P}$.

Proof. Assume the contrary. Let $d: F \rightarrow[0,1]$ be a quotient map associated with $\mathcal{Q}$ such that $d^{-1}(0)=X$. Let $s$ be the greatest lower bound of $S=\left\{r \in[0,1]: d^{-1}(r)\right.$ intersects three consecutive links of $\left.\mathcal{P}\right\}$. Since 
no element of the decomposition of $\mathrm{Cl} K$ intersects three consecutive links of $\mathscr{P}$, it follows that $s>0$. Since $\mathscr{Q}$ is continuous (Lemma 5), $s \notin S$ and $s \neq 1$.

Since $\mathscr{P}$ is free, there exist a 3-linked subchain $\mathcal{2}$ of $\mathscr{P}$ and numbers $t$, $w$ greater than $s$ such that

(1) for each number $u(t \leq u \leq w), d^{-1}(u)$ intersects each link of 2 and no element of $\mathscr{P} \backslash \mathcal{Q}$ that intersects a link of $\mathcal{Q}$.

Let $v$ be a number between $t$ and $w$.

Since $\mathscr{P}$ is regular, there exists a component $H$ of $\cup \mathscr{P}^{\prime}$ such that $H \cap d^{-1}(v) \neq \varnothing$ and

(2) $\mathrm{Cl} H$ runs straight through $\mathscr{P}$.

Since $\mathrm{Cl} H$ intersects each link of $\mathscr{P}$, the continuum $d^{-1}[[t, w]]$ does not contain $\mathrm{Cl} H$. Therefore, since $M$ is atriodic, $\mathrm{Cl} H$ intersects either $d^{-1}(t)$ or $d^{-1}(w)$. Assume without loss of generality that $\mathrm{Cl} H \cap d^{-1}(t)$ $\neq \varnothing$. By (1) and (2), there exist distinct elements $Y$ and $Z$ of the decomposition of $\mathrm{Cl} H$ such that $Y \cap d^{-1}(t) \neq \varnothing$ and $Z \cap d^{-1}(v) \neq \varnothing$. Let $I$ be the essential subcontinuum of $\mathrm{Cl} H$ that is irreducible between $Y$ and $Z$. It follows from Lemma 2 and the irreducibility of $I$ and $d^{-1}[[t, v]]$ that $I=I \cap d^{-1}[[t, v]]=d^{-1}[[t, v]]$. Since no element of the decomposition of $I$ intersects all three links of 2 , each element of the decomposition of $I$ is properly contained in an element of the decomposition of $d^{-1}[[t, v]]$. This contradicts the fact that the decomposition of $d^{-1}[[t, v]]$ is unique. Hence Lemma 16 is true.

\section{Principal results.}

THEOREM 1. Suppose $M$ is an atriodic homogeneous continuum. Suppose $N$ is an indecomposable subcontinuum of $M$ that contains a decomposable continuum. Then $M=N$. Furthermore $M$ admits a continuous decomposition $\mathscr{D}$ such that $M / \mathcal{D}$ is a solenoid and the elements of $\mathscr{D}$ are homeomorphic. Moreover if the elements of $\mathscr{Q}$ are not points, then they are tree-like hereditarily indecomposable homogeneous continua.

Proof. By the argument in paragraphs 1 and 2 in the proof of Theorem 1 of [10], $N$ has a subcontinuum $E$ of type $\lambda$. Let $k: E \rightarrow[0,1]$ be a quotient map associated with the decomposition of $E$.

Let $\varepsilon$ be a positive number.

By Lemma $13, E$ runs straight through a free normal regular chain $\rho=\left\{P_{i}: 1 \leq i \leq 5\right\}$ with the property that

(1) for each component $K$ of $\cup \mathcal{P}^{\prime}$ there is an $\varepsilon$-homeomorphism $h$ of $M$ onto $M$ such that $\mathrm{Cl} K$ is an essential subcontinuum of $h[E]$. 
For each element $Z$ of $\Omega(\mathscr{P}) \backslash \Delta(\mathscr{P})$, let $K(Z)$ be the component of $\cup \mathcal{P}^{\prime}$ that contains $Z$. Let $X(Z)$ be the end set of $\mathrm{Cl} K(Z)$ that is separated from $Z$ in $\mathrm{Cl} K(Z)$ by $P_{3}$. By Lemma 11, $\mathrm{Cl} \cup \mathcal{E}(X(Z), \mathrm{Cl} K(Z))$ is an indecomposable subcontinuum of $N$. Thus $\cup \mathcal{E}(X(Z), \mathrm{Cl} K(Z))$ intersects $P_{3} \backslash K(Z)$. Hence there is an element $F(Z)$ of $\mathcal{E}(X(Z), \mathrm{Cl} K(Z))$ that intersects $P_{3} \backslash K(Z)$. By Lemmas 7 and 16, each element of the decomposition of $F(Z)$ that intersects $\cup \mathcal{P}^{\prime}$ is an element of the decomposition of the closure of a component of $\cup \mathcal{P}^{\prime}$. Hence there is an essential subcontinuum $J(Z)$ of $F(Z)$ that contains $Z$, misses $\mathrm{Cl} P_{3}$, and has one end set in $K(Z) \cap(\cup \Delta(\mathscr{P}))$ and the other end set in $(\cup \Delta(\mathscr{P})) \backslash K(Z)$.

By Lemma 13, $J(Z)$ runs straight through a free normal regular chain $\mathscr{P}(Z)=\left\{P(Z)_{i}: 1 \leq i \leq 5\right\}$ such that

(2) $\cup \mathscr{P}(Z)$ misses $\mathrm{Cl} P_{3}$, and

(3) $\cup \Delta(\mathcal{P})$ contains $\mathrm{Cl}\left(P(Z)_{1} \cup P(Z)_{5}\right)$.

(The sets $A$ and $B$ in Lemma 13 are $\mathrm{Cl} P_{3}$ and $\cup \Delta(\mathscr{P})$, respectively.)

Let $\mathscr{B}$ be the collection of open sets $\{\cup \Delta(\mathscr{P})\} \cup\{\cup \Delta(\mathscr{P}(\mathrm{Z})): Z \in$ $\Omega(\mathscr{P}) \backslash \Delta(\mathscr{P})\}$. Since $\mathscr{P}$ is free and since $\mathscr{P}(Z)$ is free and $(3)$ holds for each element $Z$ of $\Omega(\mathscr{P}) \backslash \Delta(\mathscr{P})$, it follows that $\cup \mathscr{B}$ is a closed open subset of $M$. Hence $\Re$ covers $M$.

Let $S$ be $\cup\left(\mathcal{E}\left(k^{-1}(0), E\right) \cup \mathcal{E}\left(k^{-1}(1), E\right)\right)$.

Next we prove that

(4) $\rho(p, S)<\varepsilon$ for every point $p$ of $M$.

If $p \in \cup \Delta(\mathscr{P})$, then (4) follows from (1). Therefore we assume that $p \notin \cup \Delta(\mathscr{P})$. Let $Z$ be an element of $\Omega(\mathscr{P}) \backslash \Delta(\mathscr{P})$ such that $p \in$ $\cup \Delta(\mathscr{P}(Z))$. Let $H$ be the $p$-component of $\cup \Delta(\mathscr{P}(Z))$. Let $X$ be an end set of $\mathrm{Cl} H$. Let $K$ be the component of $\cup \mathcal{P}^{\prime}$ that contains $X$. By (1), there is an $\varepsilon$-homeomorphism $h$ of $M$ onto $M$ such that $\mathrm{Cl} K$ is an essential subcontinuum of $h[E]$. By (2) and Lemma $2, \mathrm{Cl} H$ does not intersect both $h\left[k^{-1}(0)\right]$ and $h\left[k^{-1}(1)\right]$. Hence, by Lemma $8, H$ is a subset of $T=\cup\left(\mathcal{E}\left(h\left[k^{-1}(0)\right], h[E]\right) \cup \mathcal{E}\left(h\left[k^{-1}(1)\right], h[E]\right)\right)$. Note that $T=$ $h[S]$. Since $p \in H \subset h[S]$ and $h$ is an $\varepsilon$-homeomorphism, (4) holds.

Since $\varepsilon$ may be arbitrarily small, it follows from (4) that $S$ is dense in $M$. Hence, by Lemma $10, M=N$.

For convenience we define $\mathscr{P}\left(Z_{0}\right)$ to be $\mathscr{P}$.

Since $M$ is compact, there exists a finite subcollection $e=$ $\left\{\cup \Delta\left(\mathscr{P}\left(Z_{l}\right)\right): 0 \leq i \leq n\right\}$ of $\mathscr{B}$ that covers $M$.

Let $\mathscr{Q}$ be $\cup\left\{\Delta\left(\mathscr{P}\left(Z_{i}\right)\right): 0 \leq i \leq n\right\}$.

We must show that $\mathscr{D}$ is a decomposition of $M$. Since $\mathcal{C}$ covers $M$, it suffices to show that the elements of $\mathscr{D}$ are disjoint. Let $A$ and $B$ be 
intersecting elements of $\mathscr{Q}$. If $A \cup B$ intersects $\cup \Delta(\mathscr{P})$, then, by (2), (3), and Lemma 7, $A=B$. Hence we assume that $A \cup B$ misses $\cup \Delta(\mathscr{P})$. Let $i$ and $j$ be positive integers such that $A \in \Delta\left(\mathscr{P}\left(Z_{i}\right)\right)$ and $B \in \Delta\left(\mathscr{P}\left(Z_{j}\right)\right)$. Let $I$ be the component of $\cup \mathscr{P}\left(Z_{l}\right)^{\prime}$ that contains $A$. Let $J$ be the component of $\cup \mathscr{P}\left(Z_{j}\right)^{\prime}$ that contains $B$. Since $A \cup B$ misses $\cup \Delta(\mathcal{P})$, it follows from (3) that $A \cup B$ misses both end sets of $\mathrm{Cl} I$ and both end sets of $\mathrm{Cl} J$. Thus, by Lemma 3, $A=B$. Hence $\mathcal{D}$ is a decomposition of $M$.

It follows from Lemma 14 that $\mathscr{D}$ is continuous. According to Lemma 9 , each element of $\mathscr{D}$ is either a point or a homogeneous continuum. Since $M$ is atriodic, it follows that the quotient space $M / \mathcal{O}$ is an atriodic continuum.

The quotient space $M / Q$ is homogeneous and the elements of $\mathscr{D}$ are homeomorphic. To see this first note that for each integer $i(1 \leq i \leq n)$, since $\mathscr{P}\left(Z_{l}\right)$ is a free regular chain, it follows from (2) and (3) that each component of $\cup \Delta \mathscr{P}\left(Z_{i}\right)$ misses $P_{3}$ and intersects $M \backslash \cup \mathcal{P}^{\prime}$.

Since $\mathcal{P}$ is a free regular chain, it follows from (3) and Lemma 7 that

(5) for each integer $i(0 \leq i \leq n), \Omega\left(\mathscr{P}\left(Z_{i}\right)\right) \subset \mathcal{Q}$.

By Lemma 15, for each integer $i(0 \leq i \leq n)$ there is a positive number $\varepsilon_{l}$ such that if $Z \in \Delta\left(\mathscr{P}\left(Z_{i}\right)\right)$ and $h$ is an $\varepsilon_{i}$-homeomorphism of $M$ onto $M$, then $h[Z] \in \Omega\left(\mathscr{P}\left(Z_{i}\right)\right)$. Let $\delta$ be the minimum of $\left\{\varepsilon_{l}: 0 \leq i \leq n\right\}$.

It follows from (5) that

(6) if $Z \in \mathscr{D}$ and $h$ is a $\delta$-homeomorphism of $M$ onto $M$, then $h[Z] \in \mathcal{Q}$.

Let $X$ and $Y$ be elements of $\mathscr{Q}$. Since $M$ is a continuum, there is a finite subset $\left\{x_{i}: 1 \leq i \leq m\right\}$ of $M$ such that $Y \cap W\left(x_{1}, \delta / 2\right) \neq \varnothing$, $X \cap W\left(x_{m}, \delta / 2\right) \neq \varnothing$, and $W\left(x_{i}, \delta / 2\right) \cap W\left(x_{t+1}, \delta / 2\right) \neq \varnothing$ for each integer $i(1 \leq i<m)$.

Define a set $\left\{v_{i}: 0 \leq i \leq m\right\}$ such that $v_{0} \in Y \cap W\left(x_{1}, \delta / 2\right), v_{m} \in X$ $\cap W\left(x_{m}, \delta / 2\right)$, and $v_{i} \in W\left(x_{i}, \delta / 2\right) \cap W\left(x_{l+1}, \delta / 2\right)$ for each integer $i$ $(1 \leq i<m)$. For each $i(1 \leq i \leq m)$, let $h_{i}$ be a $\delta$-homeomorphism of $M$ onto $M$ such that $h_{i}\left(v_{i}\right)=v_{i-1}$. By (6), each $h_{i}$ maps each element of $\mathscr{D}$ onto an element of $\mathscr{D}$. Therefore $h_{1} h_{2} \cdots h_{m}$ induces a homeomorphism of $M / \mathscr{D}$ onto itself that takes $X$ to $Y$. Hence $M / \mathscr{D}$ is homogeneous. Since $h_{1} h_{2} \cdots h_{m}[X]=Y$, it follows that the elements of $\mathscr{D}$ are homeomorphic.

Since $M / \mathcal{D}$ is an atriodic homogeneous continuum that contains an arc, $M / D$ is a solenoid [20, Theorem 14.4].

Suppose $\mathscr{D}$ has a nondegenerate element $Z$. By Lemma $2, Z$ is hereditarily unicoherent. Since $Z$ is homogeneous, it follows that $Z$ is indecomposable [15] [9]. In fact, $Z$ is hereditarily indecomposable; for if $Z$ 
has a decomposable subcontinuum, then, by the above argument, $M=Z$ and this is impossible. Hence $Z$ is tree-like [25]. Therefore, if the elements of $\mathscr{2}$ are not points, they are homeomorphic tree-like hereditarily indecomposable homogeneous continua.

THEOREM 2. Suppose $M$ is an atriodic homogeneous continuum that is not a solenoid and has a decomposable subcontinuum. Then $M$ admits a continuous decomposition $\mathscr{D}$ such that $M / \mathcal{D}$ is a solenoid and the elements of OD are homeomorphic tree-like hereditarily indecomposable homogeneous continua.

Proof. If $M$ is indecomposable, the conclusion follows immediately from Theorem 1. Therefore we assume that $M$ is decomposable. According to Theorem 14.7 of [20], $M$ admits a continuous decomposition $\mathscr{D}$ such that $M / \mathscr{W}$ is a simple closed curve and the elements of $\mathscr{Q}$ are homeomorphic indecomposable homogeneous continua. Let $Z$ be an element of $\mathscr{Q}$. The indecomposable continuum $Z$ is hereditarily indecomposable; for if $Z$ has a decomposable subcontinuum, it follows from Theorem 1 that $Z=M$ and this is impossible. Hence $Z$ is tree-like [25] and the proof is complete.

The following corollary to Theorem 2 answers in the affirmative Mackowiak and Tymchatyn's question [20, § 13].

COROLlaRY 1. If $M$ is an atriodic homogeneous continuum, then $M$ is 1-dimensional.

Proof. If $M$ is hereditarily indecomposable, then $M$ is tree-like [25], and, therefore, 1-dimensional. Furthermore, if $M$ is a solenoid, then $M$ is 1-dimensional. Hence we assume that $M$ is not a solenoid and has a decomposable subcontinuum. By Theorem 2, $M$ admits a continuous decomposition $\mathscr{D}$ such that $M / \mathscr{D}$ is 1-dimensional and the elements of $\mathscr{D}$ are 1-dimensional continua. According to the second inequality in the proof of Theorem VI 7 on page 92 of [14], the dimension of $M$ is either 1 or 2. Hence, by Theorem 13.4 of [20], $M$ is 1-dimensional.

COROLlARY 2. If $M$ is a tree-like atriodic homogeneous continuum, then $M$ is hereditarily indecomposable.

Proof. Suppose $M$ has a decomposable subcontinuum. By Theorem 2 , $M$ admits a monotone continuous decomposition $\mathscr{D}$ such that $M / \mathscr{D}$ is a solenoid. Since $M$ is indecomposable [15] [9], $M / \mathscr{D}$ is not a simple closed 
curve. This contradicts the fact that no tree-like continuum can be mapped onto a solenoid that is not a simple closed curve [6] [17]. Therefore $M$ is hereditarily indecomposable.

In 1968 F. B. Jones [16] suggested the following method for proving that every indecomposable homogeneous plane continuum $M$ is hereditarily indecomposable. Assume that $M$ has a decomposable subcontinuum. Find a monotone decomposition $\mathscr{D}$ of $M$ such that $M / \mathscr{D}$ is a homogeneous plane continuum that contains an arc. It follows from a theorem of Bing [4] that $M / O$ is a simple closed curve and this contradicts the fact that $M$ is indecomposable.

Every indecomposable homogeneous plane continuum is atriodic [10, Lemma 1]. In Theorem 2 (above), since the elements of $\mathscr{Q}$ are tree-like, if $M$ is planar, then $M / \mathscr{Q}$ is planar [21]. Hence Theorem 2 provides the decomposition that Jones requested and we obtain the following:

COROLlARY 3. Every indecomposable homogeneous plane continuum is hereditarily indecomposable [11].

ACKNOWLEDGEMENT. The author is indebted to Wallace Etterbeek and Roger Leezer of the CSUS School of Arts and Sciences for granting released time to prepare this manuscript and to Sukhjit Singh for several helpful conversations about atriodic homogeneous continua.

\section{REFERENCES}

[1] R. H. Bing, A homogeneous indecomposable plane continuum, Duke Math. J., 15 (1948), 729-742.

[2] Higher-dimensional hereditarily indecomposable continua, Trans. Amer. Math. Soc., 71 (1951), 267-273.

[3] __ Each homogeneous nondegenerate chainable continuum is a pseudo-arc, Proc. Amer. Math. Soc., 10 (1959), 345-346.

[4] A simple closed curve is the only homogeneous bounded plane continuum that contains an arc, Canad. J. Math., 12 (1960), 209-230.

[5] R. H. Bing and F. B. Jones, Another homogeneous plane continuum, Trans. Amer. Math. Soc., 90 (1959), 171-192.

[6] K. Borsuk, On movable compacta, Fund. Math., 66 (1969), 137-146.

[7] C. E. Burgess, Homogeneous 1-dimensional continua, General Topology and Modern Analysis, Academic Press, New York, 1981, 169-175.

[8] E. G. Effros, Transformation groups and $C^{*}$-algebras, Ann. of Math., 81 (1965), 38-55.

[9] G. R. Gordh, Jr., On homogeneous hereditarily unicoherent continua, Proc. Amer. Math. Soc., 51 (1975), 198-202.

[10] C. L. Hagopian, Homogeneous plane continua, Houston J. Math., 1 (1975), 35-41.

[11] In Indecomposable homogeneous plane continua are hereditarily indecomposable, Trans. Amer. Math. Soc., 224 (1976), 339-350.

[12] _ A characterization of solenoids, Pacific J. Math., 68 (1977), 425-435. 
[13] C. L. Hagopian and J. T. Rogers, Jr., A classification of homogeneous circle-like continua, Houston J. Math., 3 (1977), 471-474.

[14] W. Hurewicz and H. Wallman, Dimension Theory, Princeton University Press, Princeton, N. J., 1948.

[15] F. B. Jones, Certain homogeneous unicoherent indecomposable continua, Proc. Amer. Math. Soc., 2 (1951), 855-859.

[16] Homogeneous plane continua, Proc. Auburn Topology Conf., Auburn University, Auburn, Ala., (1969), 46-56.

[17] J. Krasinkiewicz, Curves which are continuous images of tree-like continua are movable, Fund. Math., 89 (1975), 233-260.

[18] K. Kuratowski, Theorie des continus irreductible entre deux points II, Fund. Math., 10 (1927), 225-276.

[19] Topology, Volume 2, 3rd ed., Monografie Mat., TOM 21, PWN, Warsaw, 1961; English translation, Academic Press, New York; PWN, Warsaw, 1968.

[20] T. Mackowiak and E. D. Tymchatyn, Continuous mappings on continua II, Dissertationes Math., 225 (1983).

[21] R. L. Moore, Concerning upper semi-continuous collections of continua, Trans. Amer. Math. Soc., 27 (1925), 416-428.

[22] __ Foundations of point set theory, rev. ed., Amer. Math. Soc. Colloq. Publ., vol. 13, Amer. Math. Soc., Providence, R. I., 1962.

[23] J. T. Rogers, Jr., Solenoids of pseudo-arcs, Houston J. Math., 3 (1977), 531-537.

[24] _ Decompositions of homogeneous continua, Pacific J. Math., 99 (1982), 137-144.

[25] Homogeneous, hereditarily indecomposable continua are tree-like, Houston J. Math., 8 (1982), 421-428.

[26] E. S. Thomas, Jr., Monotone decompositions of irreducible continua, Rozprawy Matematyczne, 50 (1966), 1-74.

Received September 17, 1982. The author was partially supported by NSF Grant MCS8205282 .

California State University

SACRAMENTO, CA 95819 



\section{PACIFIC JOURNAL OF MATHEMATICS \\ EDITORS}

Donald BaBBITT (Managing Editor)

University of California

Los Angeles, CA 90024

Hugo RossI

University of Utah

Salt Lake City, UT 84112

C. C. Moore and Arthur Ogus

University of California

Berkeley, CA 94720
J. DugundiI

Department of Mathematics

University of Southern California

Los Angeles, CA 90089-1113

R. FINN and H. SAMELSON

Stanford University

Stanford, CA 94305

\section{ASSOCIATE EDITORS}
R. ARENS
E. F. BECKENBACH
B. H. NeUMANN
F. WOLF
K. YoshidA (1906-1982)

\section{SUPPORTING INSTITUTIONS}

\author{
UNIVERSITY OF ARIZONA \\ UNIVERSITY OF BRITISH COLUMBIA \\ CALIFORNIA INSTITUTE OF TECHNOLOGY \\ UNIVERSITY OF CALIFORNIA \\ MONTANA STATE UNIVERSITY \\ UNIVERSITY OF NEVADA, RENO \\ NEW MEXICO STATE UNIVERSITY \\ OREGON STATE UNIVERSITY
}

\author{
UNIVERSITY OF OREGON \\ UNIVERSITY OF SOUTHERN CALIFORNIA \\ STANFORD UNIVERSITY \\ UNIVERSITY OF HAWAII \\ UNIVERSITY OF TOKYO \\ UNIVERSITY OF UTAH \\ WASHINGTON STATE UNIVERSITY \\ UNIVERSITY OF WASHINGTON
}

The Supporting Institutions listed above contribute to the cost of publication of this Journal, but they are not owners or publishers and have no responsibility for its content or policies.

Mathematical papers intended for publication in the Pacific Journal of Mathematics should be in typed form or offset-reproduced (not dittoed), double spaced with large margins. Please do not use built up fractions in the text of the manuscript. However, you may use them in the displayed equations. Underline Greek letters in red, German in green, and script in blue. The first paragraph must be capable of being used separately as a synopsis of the entire paper. In particular it should contain no bibliographic references. Please propose a heading for the odd numbered pages of less than 35 characters. Manuscripts, in triplicate, may be sent to any one of the editors. Please classify according to the scheme of Math. Reviews, Index to Vol. 39. Supply name and address of author to whom proofs should be sent. All other communications should be addressed to the managing editor, or Elaine Barth, University of California, Los Angeles, California 90024.

There are page-charges associated with articles appearing in the Pacific Journal of Mathematics. These charges are expected to be paid by the author's University, Government Agency or Company. If the author or authors do not have access to such Institutional support these charges are waived. Single authors will receive 50 free reprints; joint authors will receive a total of 100 free reprints. Additional copies may be obtained at cost in multiples of 50 .

The Pacific Journal of Mathematics is issued monthly as of January 1966. Regular subscription rate: $\$ 132.00$ a year (6 Vol., 12 issues). Special rate: $\$ 66.00$ a year to individual members of supporting institutions.

Subscriptions, orders for numbers issued in the last three calendar years, and changes of address should be sent to Pacific Journal of Mathematics, P.O. Box 969, Carmel Valley, CA 93924, U.S.A. Old back numbers obtainable from Kraus Periodicals Co., Route 100, Millwood, NY 10546.

The Pacific Journal of Mathematics ISSN 0030-8730 is published monthly by the Pacific Journal of Mathematics at P.O. Box 969, Carmel Valley, CA 93924. Application to mail at Second-class postage rates is pending at Carmel Valley, California, and additional mailing offices. Postmaster: Send address changes to Pacific Journal of Mathematics, P. O. Box 969, Carmel Valley, CA 93924.

PUBLISHED BY PACIFIC JOURNAL OF MATHEMATICS, A NON-PROFIT CORPORATION

Copyright $\odot 1984$ by Pacific Journal of Mathematics 


\section{Pacific Journal of Mathematics}

\section{Vol. 113, No. $2 \quad$ April, 1984}

Alan Adolphson, On the Dwork trace formula ...................257

Amos Altshuler and Leon Steinberg, Enumeration of the quasisimplicial

3 -spheres and 4-polytopes with eight vertices .................. 269

Kenneth R. Goodearl, Cancellation of low-rank vector bundles .......... 289

Gary Fred Gruenhage, Ernest A. Michael and Yoshio Tanaka, Spaces

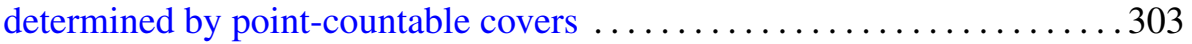

Charles Lemuel Hagopian, Atriodic homogeneous continua .......... 333

David Harbater, Ordinary and supersingular covers in characteristic $p$. . . 349

Domingo Antonio Herrero, Continuity of spectral functions and the lakes

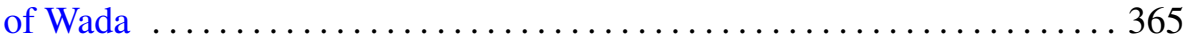

Donald William Kahn, Differentiable approximations to homotopy resolutions and framed cobordism ....................... 373

K. McGovern, On the lifting theory of finite groups of Lie type $\ldots \ldots \ldots . \ldots 383$

C. David (Carl) Minda, The modulus of a doubly connected region and the geodesic curvature-area method ............................. 395

Takuo Miwa, Complexes are spaces with a $\sigma$-almost locally finite base $\ldots . .407$

Ho Kuen Ng, Finitely presented dimension of commutative rings and modules

Roger David Nussbaum, A folk theorem in the spectral theory of

$C_{0}$-semigroups

J. S. Okon, Prime divisors, analytic spread and filtrations

Harold Raymond Parks, Regularity of solutions to elliptic isoperimetric problems

R. Sitaramachandra Rao and M. V. Subba Rao, Transformation formulae for multiple series

Daniel Ruberman, Imbedding punctured lens spaces and connected sums

Uri Srebro, Deficiencies of immersions 